\title{
An Investigation of Makeup Ingredients and Their Effects on Acne Cosmetica with Dermatologic Practice Recommendations
}

\author{
Hira Ghani, BA ${ }^{1}$, Raphia K. Rahman BA, MBS${ }^{2}$, Kevin Liu, DO ${ }^{3}$, Stefani Cubelli, DO ${ }^{4}$ \\ ${ }^{1}$ New York Institute of Technology College of Osteopathic Medicine, Old Westbury, NY \\ 2 Rowan School of Osteopathic Medicine, Rowan, NJ \\ ${ }^{3}$ Lehigh Valley Dermatology, Allentown, PA \\ ${ }^{4}$ St. John's Episcopal Hospital, Far Rockaway, NY
}

\section{ABSTRACT}

Acne cosmetica, a type of acne linked to cosmetic usage, is characterized by persistent mild breakouts and occurs due to the interplay between sebum and trapped comedogenic products in makeup products. Regular cosmetic usage may cause acne since it has been determined that a reduction in makeup application can help reduce its severity. There is a pressing need for dermatologists and patients to select appropriate, well-tolerated, and non-comedogenic makeup products containing active ingredients that help to eradicate acne. This literature review will thus examine common ingredients found in makeup products that act as comedogenic irritants, acnefriendly ingredients, and explore dermatologic recommendations to address beauty product use in acne-prone patients. Both PubMed and Google Scholar were searched using keywords skincare and makeup ingredients combined with acne cosmetica in adolescents and dermatology. This literature review has indicated that patients suffering from acne should be recommended to avoid using comedogenic products and switch to acne-friendly ingredients that are safe for skin. Dermatologists should recommend the use of prescription topical medications containing ingredients such as retinol and salicylic acid to yield visible and noticeable results. These findings help to strengthen the dermatologists' understanding of common active ingredients found in beauty products and helps guide recommendations for patients suffering from acne.

\section{INTRODUCTION}

Acne vulgaris is the most common skin disease affecting late adolescents across the globe. According to a Global Burden of Disease study, approximately $85 \%$ of young adults between the ages of 12-25 are afflicted by acne vulgaris. Its prevalence has also continued to increase over time in the US as well as other western countries including France and the United Kingdom. ${ }^{1}$ The prevalence of adolescent acne has been reported to range between 81 to 95 percent in males and 79 to 82 percent in females. ${ }^{2}$ The annual treatment cost of acne is approximately $\$ 3$ billion. ${ }^{3}$ Given the rise of acne diagnosis not only in teenagers but also across multiple nationalities and races, it is important for clinicians to understand the basic pathogenesis, and most importantly, investigate new exacerbating factors that may not have been previously associated with acne epidemiology.

Acne is a chronic inflammatory skin disorder of the sebaceous glands of the hair follicle. It is caused by a cascade of different factors, subsequently leading to the formation of a microcomedone, and eventually acne September 2021 Volume 5 Issue 5 
vulgaris. ${ }^{4}$ The most common cause of acne vulgaris is an increase of sebum production and secretion, or a change in sebum components, which results in the clogging of pores. ${ }^{4}$ Excessive sebum production is caused by the enlargement of sebaceous glands due increasing levels of DHEA-S during the prepubertal period. This makes the skin appear shiny and eventually leads to the formation of a hardened plug or comedone. ${ }^{5}$ Acne can also be triggered by a change in sebum composition. Acne is specifically triggered when there is an imbalance between the ratio of monounsaturated fatty acids to total fatty acids. The ratio of triglycerides and wax esters is notably higher in the sebum of individuals with acne. ${ }^{4}$

Acne vulgaris can further be subdivided into non-inflammatory and inflammatory acne. Non-inflammatory acne is composed of whiteheads (open comedones) and blackheads (closed comedones) while inflammatory acne is associated with painful red cysts that often cause scarring over time. Hormonal imbalance triggers the excessive production of sebum, which when combined with dead skin cells, promotes the development of both inflammatory and noninflammatory acne. ${ }^{2}$ Propionibacterium acnes, a skin-colonizing bacteria, is highly responsible for inducing inflammatory acne by increasing sebum secretion and leading to a mounted immune response via increased expression of pro-inflammatory mediators such as IL-1 alpha, IL-2, and most importantly, TLR-2. ${ }^{6}$

Moreover, a high glycemic index of diet may also participate in the pathogenesis of both inflammatory and non-inflammatory types of acne vulgaris. Diets based on products with a high glycemic index leads to hyperinsulinemia, thus stimulating the secretion of androgens and causing an increased production of sebum by elevating the level of insulin-like growth factor 1 (IGF1). ${ }^{7}$ IGF-1 influences comedogenic factors such as androgens, growth hormone and glucocorticoids. ${ }^{7}$ These comedogenic factors increase the endogenous levels of IGF-1 in blood, which further increases levels of androgens, thereby creating a vicious cycle of excessive production of sebum. ${ }^{7}$

In today's world, popular retail stores such as Sephora $\AA$ and Ulta Beauty ${ }^{\circledR}$ have lured customers into purchasing various high end and luxurious makeup products; the 2019 revenue of the cosmetic and beauty industry in the U.S. was approximately 49.2 billion dollars alone. ${ }^{8}$ However, these products may often contain ingredients that are potentially harmful to the skin, especially if the consumer has acne-prone or sensitive skin. Acne cosmetica is a form of acne linked to cosmetic usage, characterized by persistent mild breakouts resulting from the complex interplay between sebum and trapped comedogenic products in makeup. ${ }^{9}$ While makeup can damage the skin if used aggressively and routinely, noncomedogenic makeup applied with caution and in moderate amounts may conceal scars and blemishes, helping to increase the self-esteem of adolescents struggling with acne. ${ }^{10}$ The purpose of this literature review is to examine ingredients found in makeup products that can act as irritants and as an agent of camouflage for acne. Furthermore, this literature review will investigate whether makeup is recommended for use on acneprone skin, review acne-friendly ingredients found in products, and propose recommendations on how dermatologists should counsel acne-prone patients in regards to product choices for makeup application. 


\section{METHODS}

We searched PubMed and Google Scholar using search terms skincare and makeup ingredients combined with acne cosmetica in adolescents and dermatology. We limited our search to articles available in English and those published between 1972 and 2020.

\section{DISCUSSION}

\section{Acne and Cosmetics}

For many years, it has been speculated that cosmetic products are implicated in the pathogenesis of acne vulgaris. Acne cosmetica, as the name suggests, is one such form of acne linked to cosmetic usage. The complex interplay between sebum and trapped comedogenic products in makeup cause the eruption of acne cosmetica, which is characterized by persistent mild breakouts, comprising comedones along the forehead, cheeks, neck and scalp. ${ }^{9}$ Occasionally, papules and pustules may also be seen in acne cosmetica, but they are far less common. ${ }^{9}$ However, this association was difficult to prove as both the incidence of acne vulgaris and the use of cosmetics have steadily increased over time.

Recently, there have been an increasing amount of studies linking cosmetic usage to the development of acne as well as the subsequent severity of acne. For example, a study from Brazil demonstrated 45 percent of women who used makeup daily had a distinguished dermatosis. Approximately 14 percent of these patients suffered specifically from acne cosmetica. ${ }^{11}$ Similarly, a study by Perera et al was conducted to determine the effect of makeup usage on acne severity. In their study of 140 adolescent girls from 3 different schools in Colombo, Sri Lanka, a statistically significant correlation was demonstrated between cosmetic exposure and acne grade. Only $8.6 \%$ of the study population was free of acne (grade 0 ), whereas the remaining majority (91.4\%) obtained a high intensity acne grading. These results suggest that regular cosmetic usage might be a causative factor for acne in post-pubertal girls, and reduction in makeup application may reduce the severity of acne. ${ }^{12}$ However, there is increasing interest in cosmetic products as components of acne patients' overall management plans, which can complement the medical regimen. ${ }^{13}$ In females, in particular, there is a need for cosmetic products that can effectively cover the signs of this highly visible skin condition to reduce the emotional impact of the disease. Use of cosmetics can also increase acne patients' adherence with their medical regimen, which is estimated to be poor in $50 \%$ of patients. ${ }^{14}$ Therefore, we see that people with acne tend to use makeup more often to cover their blemishes while also taking medications that help subside acne.

\section{Ingredients and Vehicle of Makeup}

Traditionally, researchers and dermatologists have advised individuals with oily or acne-prone skin to refrain from using moisturizers, foundations and other personal care items consisting of comedogenic or oilbased products. These ingredients may lead to the clogging of pores and ultimately to the formation of acne. Similarly, acne patients have been advised to refrain from products that are thick and creamy in consistency as they may also clog pores. Unfortunately, even the use of a robust facial cleanser, face wash or makeup removing wipes has little to no effect on controlling acne if comedogenic makeup is used routinely. ${ }^{15}$ We also suggest dermatologists to advise 
patients to make sure to wash all makeup application accessories and tools, including brushes, beauty blenders and powder puffs frequently with a mild non-comedogenic cleanser or face wash and allowing them to air dry. Clean makeup application brushes will ensure minimal clogging of pores that usually results from layers of old makeup products embedded in these brushes.

\section{The Comedogenic scale}

Comedogenicity indicates the potential to lead to formation of comedones and development of acne. Finished products containing high comedogenicity ingredients should be evaluated for their comedogenic potential. In an effort to better understand the effect of makeup ingredients and their future acne forming capabilities, the comedogenic scale was devised. The comedogenic scale rates the active ingredients of different makeup and skincare formulations based on their propensity to clog pores. The scale uses a numbering system of 0 to 5 , with 0 being the lowest and 5 being the highest. ${ }^{16}$

The Comedogenic scale:

0 - won't clog pores at all

1 - very low likelihood of clogging pores

2 - moderately low likelihood

3 - moderate likelihood

4 - fairly high likelihood

5 - high likelihood of clogging pores

Non-comedogenic products that do not clog pores have a comedogenic rating of 2 or less. Makeup with a moderate (3) to high comedogenic (5) score should be avoided in teens with acne prone skin as it greatly increases the probability of an acne breakout or the worsening of acne in those who have acne prone skin. ${ }^{16}$ Some common makeup ingredients implicated in the pathogenesis of acne are listed below along with their comedogenicity.

\section{Algae Extract}

Algae extract can be found in some concealers and moisturizers. It is rated as 5 on the comedogenic scale. Products containing algae extract should be avoided in acne because of its high tendency to clog pores and irritate the skin, causing redness and itching. ${ }^{17}$

\section{Benzaldehyde}

Benzaldehyde is an added fragrance used in cosmetics and skincare products. It is rated as 3.5 on the comedogenic scale and leads to clogged pores and irritated skin. This effect may be seen with other fragrances commonly used in cosmetics and skin care products. ${ }^{18}$

\section{D\&C Red}

D \& C red is a dye added to give color to cosmetics. This dye is commonly found in blushes or bronzers, which may explain the predominance of cosmetic acne in the cheekbone area. ${ }^{16}$ This will be seen as ' $D$ \& $C$ Red' on the label and followed by a number. Numbers 27 and 40 are rated high on the scale, whereas the others are rated as medium on the scale. Interestingly, the vehicle carrying D\&C determines its comedogenic grade. For instance, polyethylene glycol is less comedogenic than D\&C red when incorporated into other vehicles such as mineral oil. ${ }^{19}$

\section{Silicones}

There are numerous silicone variations found in beauty products, including but not limited to methicone, dimethicone, trimethicone, and cyclomethicone. These 
ingredients create a silky, smooth feel and are often found in cosmetic primers, deodorants, sunscreens, and leave-in hair styling products. Sensitive and acne-prone skin can end up with breakouts if silicone is used regularly, since it may lead to the clogging of pores. ${ }^{20}$

\section{Coconut and Avocado Oil}

Both coconut and avocado oil have long been used as a "natural" alternative for providing skin and hair with a radiant glow, but they are responsible for the formation of comedones due to their occlusive properties. Moreover, these oils are both difficult to remove, thus adding to their occlusive and pore-clogging nature. These oils have a high comedogenic index of 3-4. ${ }^{19}$

\section{Isopropyl Isostearate}

Isopropyl isostearate is an emollient found in many lotions and skin care products. It is ranked the highest on the comedogenic scale with a score of 5. Isopropyl isostearate is the ester of isopropyl alcohol (rubbing alcohol) and stearic acid (a thickening agent) and is most commonly found in skin care products and eye cosmetics. ${ }^{20}$

\section{Products for Acne Prone Skin}

Thankfully, many moisturizers and cosmetics are now being designed for acne prone skin types. Specifically, these products incorporate non-comedogenic ingredients along with a water base that can provide full coverage to acne lesions and scars without causing a detrimental effect to the skin by aggravating breakouts by the blockage of pores. ${ }^{21}$ Some of these makeup products also contain ingredients aimed at treating acne such as salicylic acid. In general, it is recommended to use an oil-free makeup with light consistency to prevent breakouts. Some other key ingredients patients may benefit from in their cosmetic products include hyaluronic acid, retinol, LAscorbic Acid, sulfur and minerals. ${ }^{22}$ Below, we have included a list of studied ingredients that are helpful in alleviating as well as preventing acne onset.

\section{Salicylic Acid}

Salicylic acid is lipophilic acid with beta hydroxyl acid capabilities. It has played a role in acne prevention for many years and has gained popularity as the main ingredient in many commercially available facial cleansers. Salicylic acid is less irritating than skin care products containing alpha-hydroxy acids, while providing similar improvement in skin texture and color. The ability of salicylic acid to exfoliate the stratum corneum along with its comedolytic property makes it a useful peeling agent for acne. Salicylic acid is known to disrupt cellular junctionsdesmosomes, without lysing intercellular keratin filaments. ${ }^{23}$ Given the accessibility of salicylic acid, there are many over the counter skin care products that may be beneficial for those with acne prone skin.

\section{Hyaluronic Acid}

Hyaluronic acid (HA), a glycosaminoglycan, is known for its tendency to keep skin plump and supple due to its humectant capabilities while preventing acne breakouts. Hyaluronic acid plays a vital role in the synthesis of extracellular matrix molecules and epidermal cell interaction with the surrounding environment. It modulates cellular immunity by preventing bacterial infections and halting allergic reactions. Its most important property is to hydrate the skin by holding large amounts of moisture in the dermis. Hyaluronic acid is a component of the body's connective tissues, and is known to cushion and lubricate. With 
aging, however, the amount of hyaluronic acid found naturally within the dermis decreases to about five percent of baseline. ${ }^{24}$ Skin care products often couple hyaluronic acid with vitamin $C$ products to assist in penetration.

\section{Retinol}

Retinol is derived from vitamin $\mathrm{A}$ and is increasing in popularity in many over-thecounter skin care products aimed at acne as well as "anti-aging". Retinol has a molecular structure small enough to penetrate the skin to the deep layers to interact with collagen and elastin. Retinol also binds to specific proteins in the nucleus of skin cells which allows it to influence the transcription of many genes and transcription factors. ${ }^{25}$ The overall effect of retinol includes, but is not limited to, correction of skin pigmentation, a decrease in the appearance of fine lines and wrinkles due to increased collagen synthesis, and reduction of sun damage and inflammation. Cosmetic products may also use retinyl palmitate which is closely related to retinol, but is less potent. Topical retinoids, as monotherapy, are mainly used in patients with noninflammatory comedones, in combination with other topical and systemic drugs in mild, moderate, and severe inflammatory acne, and also as a maintenance treatment when oral treatment is stopped. The following retinoid molecules are used today in the topical management of acne: tretinoin (alltrans retinoic acid), isotretinoin (13-cis isomer), adapalene, tazarotene, and retinaldehyde. ${ }^{25}$

\section{L-Ascorbic Acid}

L-ascorbic acid (LAA) is the chemically active form of Vitamin C. Vitamin C is a potent antioxidant that can be used topically to treat and prevent changes associated with photo aging. It can also be used for the treatment of hyper-pigmentation resulting from acne scars. L-Ascorbic acid interacts with copper ions at the tyrosinase-active site and inhibits action of the enzyme tyrosinase, thereby decreasing the melanin formation resulting in decreased hyperpigmentation. Vitamin C has also been shown to stimulate the synthesis of collagen by stimulating lipid peroxidation. The by-product of this stimulation, malondialdehyde, in turn stimulates collagen gene expression. Vitamin C also directly activates the transcription of collagen synthesis and stabilizes pro-collagen mRNA, thereby regulating collagen synthesis. ${ }^{26}$

\section{Sulfur or Sulfacetamide}

Sodium sulfacetamide 10\%-sulfur 5\% (SSS) emollient in face wash and cleanser works by stopping the growth of certain bacteria on skin including Propionibacterium acnes. It is also known to exhibit moisturizing properties. In addition, SSS emollient foam has been shown to markedly reduce colony counts of Propionibacterium acnes in vitro. ${ }^{27}$

\section{A Summary of Dermatologic Recommendations for Acne Cosmetica}

Although it is best to keep acne prone skin bare as much as possible, patients may feel the pressure or desire to conceal their lesions. This is achievable with the right choice of skincare and beauty products such as foundation and concealer, along with mastery in makeup application techniques.

However, we suggest it is beneficial for the dermatologist to advise patients to make sure to frequently wash all makeup application accessories and tools, including brushes, beauty blenders and powder puffs with a mild non-comedogenic cleanser or face wash. While there are many products 
available on the market to combat acne vulgaris, a robust understanding of the active ingredients in skincare and beauty products is necessary to help patients struggling with acne vulgaris. In short, patients with acne should avoid product ingredients such as avocado and coconut oil, silicones, isopropyl isostearate, D\&C Red, benzaldehyde and algae extract for skin use. Instead, it is recommended that products containing ingredients such as LAscorbic acid, hyaluronic acid, salicylic acid, retinol, and sulfur be used in acne-prone patients to help reduce inflammation.

\section{CONCLUSION}

In conclusion, there are various advantages and disadvantages associated with makeup use in acne. Makeup can damage sensitive and acne-prone skin if used daily and excessively However, the use of dermatologist enforced non-comedogenic makeup may help conceal acne induced blemishes, and therefore boost selfconfidence in adolescents who may otherwise feel uncomfortable going out with bare skin. There is a pressing need for dermatologists and patients to select appropriate, well-tolerated, and noncomedogenic skin care and makeup products containing active ingredients that help to eradicate acne. We recommend that acne-prone individuals consult dermatologists prior to buying makeup and skincare products to help determine whether product ingredients are safe to use with no risk of causing skin inflammation, and to also determine its efficacy in improving skin conditions. Finally, dermatologists should emphasize the importance of utilizing prescription topical medications consisting of key dermatocosmetic ingredients that control acne, such as retinol and salicylic acid, so that patients with acne can notice visible results over time.

Conflict of Interest Disclosures: None

Funding: None

Corresponding Author:

Hira Ghani

940 N New St.

Bethlehem, PA 18018

Phone: 201-925-5165

Email: hghani01@nyit.edu

\section{References:}

1. Lynn DD, Umari T, Dunnick CA, Dellavalle RP. The epidemiology of acne vulgaris in late adolescence. Adolesc Health Med Ther. 2016;7:13-25.

2. Skroza N, Tolino E, Mambrin A, et al. Adult Acne Versus Adolescent Acne: A Retrospective Study of 1,167 Patients. The Journal of clinical and aesthetic dermatology. 2018;11(1):21-25.

3. Wischhover C. A Dermatologist Explains All the Ways to Treat Acne. Racked Website. www.racked.com/2018/4/12/17179550/popularacne-treatments-dermatologist-aad. Accessed November 20, 2020.

4. Li X, He Congfen, Chen Z, Zhou C, Gan Y, Jia Y. A review of the role of sebum in the mechanism of acne pathogenesis. Journal of Cosmetic Dermatology. 2017;16(2):168-173.

5. Zaenglein AL, Pathy AL, Schlosser BJ, et al. Guidelines of care for the management of acne vulgaris. J Am Acad Dermatol. 2016;74:945.

6. Tanghetti EA. The role of inflammation in the pathology of acne. J Clin Aesthet Dermatol. 2013;6(9):27-35.

7. Kucharska A, Szmurło A, Sińska B. Significance of diet in treated and untreated acne vulgaris. Postepy Dermatol Alergol. 2016;33(2):81-86.

8. Revenue of the cosmetic and beauty industry in the United States from 2002 to 2020. Statista website.

www.statista.com/statistics/243742/revenue-ofthe-cosmetic-industry-in-the-

us/\#: :text=The\%20revenue\%20of\%20the\%20U. S.,billion\%20U.S.\%20dollars\%20in\%202019.\&text =Since $\% 20$ the $\% 20$ early $\% 20$ twentieth $\% 20$ century, handful\%20of\%20multi-national\%20corporations. Accessed December 24, 2020.

9. Kligman AM, Mills OH. Acne cosmetica. Arch Dermatol. 1972 Dec;106(6):843-850. 
10. Murakami-Yoneda $Y$, Hata M, Shirahige $Y$, Nakai $\mathrm{K}$, Kubota Y. Effects of Makeup Application on Diverting the Gaze of Others from Areas of Inflammatory Lesions in Patients with Acne Vulgaris. Journal of Cosmetics, Dermatological Sciences and Applications. 2015;5(2):134-141.

11. Alvarez Manny. The damaging effects makeup can have on teens. New York Post Website. https://nypost.com/2017/06/17/the-damagingeffects-makeup-can-have-on-teens/. Accessed November 12, 2020.

12. Perera MPN, Peiris WMDM, Pathmanathan D, Mallawaarachchi S, Karunathilake IM. Relationship between acne vulgaris and cosmetic usage in Sri Lankan urban adolescent females. $J$ Cosmet Dermatol. 2018;17(3):431-436.

13. Del Rosso JQ. The role of skin care as an integral component in the management of acne vulgaris: part 1: the importance of cleanser and moisturizer ingredients, design, and product selection. J Clin Aesthet Dermatol. 2013;6(12):19-27.

14. Dreno B, Thiboutot D, Gollnick H, et al. Largescale worldwide observational study of adherence with acne therapy. Int J Dermatol. 2010;49(4):448-456.

15. Cichowski Heather. Watch Out for These Skin Care Ingredients if You Have Acne-Prone Skin. The Fashion Spot Website. www.thefashionspot.com/beauty/787783ingredients-that-cause-acne. Accessed October 29, 2020.

16. Fulton JE Jr, Pay SR, Fulton JE 3rd. Comedogenicity of current therapeutic products, cosmetics, and ingredients in the rabbit ear. $J A m$ Acad Dermatol. 1984 Jan;10(1):96-105.

17. Narayan V. Holistic Skincare and Selection of Skincare Products in acne. Archives of Clinical \& Experimental Dermatology. 2020; 2(1);1-3.

18. Maharani A, Pratiwi W, Nauphar D. Changing Cosmetic Brands Increase Risk Of Frequency And Degree Of Acne Vulgaris In Female Undergraduate Students. Proceedings of International Conference on Applied Science and Health. 2018; 3:53-57.

19. Rubin I, Maged G, Garruto J, McCarver B. Noncomedogenic and non-acnegenic hair and scalp care formulations and method for use. www.patents.google.com/patent/US9949915B2/en . Accessed December 13, 2020.

20. Simion, FA. Acnegenicity and comedogenicity testing for cosmetics. Handbook of Cosmetic Science and Technology. 2001; 837-844.

21. Chularojanamontri L, Tuchinda P, Kulthanan K, Pongparit K. Moisturizers for Acne: What are their Constituents? J Clin Aesthet Dermatol. 2014;7(5):36-44.
22. Reszko Anetta E, Berson Diane, Lupo Mary P. Cosmeceuticals: practical applications. Dermatol Clin. 2009;27(4):401-416.

23. Arif T. Salicylic acid as a peeling agent: a comprehensive review. Clin Cosmet Investig Dermatol. 2015;8:455-461.

24. Jegasothy SM, Zabolotniaia V, Bielfeldt S. Efficacy of a New Topical Nano-hyaluronic Acid in Humans. J Clin Aesthet Dermatol. 2014;7(3):2729.

25. Rigopoulos D, loannides D, Kalogeromitros D, Katsambas AD. Comparison of topical retinoids in the treatment of acne. Clin Dermatol. 2004;22(5):408-411.

26. Telang PS. Vitamin $C$ in dermatology. Indian Dermatol Online J. 2013;4(2):143-146.

27. Del Rosso JQ. The use of sodium sulfacetamide $10 \%$-sulfur $5 \%$ emollient foam in the treatment of acne vulgaris. J Clin Aesthet Dermatol. 2009;2(8):26-29. 